\title{
Tuning the Magnetic Vortex State in Magnetite Nanodiscs for Remote Control of Biological Signalling
}

Danijela Gregurec ${ }^{1,2, \#}$, Alexander W. Senko ${ }^{1,3 \#}$, Andrey Chuvilin ${ }^{4,5}$, Pooja D. Reddy ${ }^{3}$, Ashwin Sankararaman $^{1}$, Dekel Rosenfeld ${ }^{1,2}$, Po-Han Chiang ${ }^{1,2}$, Francisco Garcia ${ }^{2,3}$, Ian Tafel ${ }^{6}$, Georgios Varnavides $^{1,3,7}$ Eugenia Ciocan $^{8}$, Polina Anikeeva ${ }^{1,2,3,9 *}$

${ }^{1}$ Research Laboratory of Electronics, Massachusetts Institute of Technology, Cambridge, MA

${ }^{2}$ McGovern Institute for Brain Research, Massachusetts Institute of Technology, Cambridge, MA

${ }^{3}$ Department of Materials Science and Engineering, Massachusetts Institute of Technology, Cambridge, MA

${ }^{4}$ CIC nanoGUNE, San Sebastián, Spain

${ }^{5}$ Ikerbasque, Basque Foundation for science, Bilbao, Spain

${ }^{6}$ Department of Neurosurgery, Brigham and Women's Hospital, Boston, MA

${ }^{7}$ John A. Paulson School of Engineering and Applied Sciences, Harvard University, Cambridge, MA

${ }^{8}$ Department of Engineering and Physical Sciences, Bunker Hill Community College, Boston, MA

${ }^{9}$ Department of Brain and Cognitive Sciences, Massachusetts Institute of Technology, Cambridge, MA

*All correspondence and requests for samples should be addressed to anikeeva@mit.edu \#Authors contributed equally to this manuscript 


\begin{abstract}
When a vortex configuration of spins is present in magnetic nanomaterials, it enables rapid control over their magnetization direction and magnitude. The vortex configuration manifests in near zero net magnetic moment in the absence of a magnetic field, affording greater colloidal stability of magnetic nanomaterials in suspensions. Together, these properties invite the application of magnetic vortex particles as transducers of externally applied minimally invasive magnetic stimuli in biological systems. Using magnetic modelling and electron holography, we predict and experimentally demonstrate magnetic vortex states in an array of colloidally synthesized magnetite nanodiscs 98-226 $\mathrm{nm}$ in diameter. The magnetic nanodiscs were applied as transducers of torque for remote control of mechanosensory cells in weak $(\leq 28 \mathrm{mT})$, slowly varying $(\leq 5 \mathrm{~Hz})$ magnetic fields. The extent of cellular response was determined by the magnetic nanodisc volume and magnetic field conditions. With their high-throughput colloidal synthesis and tunable dimensions, magnetic vortex particles may find applications as switches in engineering and biomedical systems.
\end{abstract}

\title{
Main
}

Magnetic particles with dimensions near the single-to-multi-domain transition can exhibit a magnetic vortex ground state, where magnetic flux closure is achieved without domain walls (Fig. 1). The vortex configuration is more likely to occur in disc-shaped particles made of magnetic materials with high saturation magnetization, which drives the minimization of the magnetostatic energy, and low magnetocrystalline anisotropy and exchange stiffness, which allows for a circular configuration of spins without large energy penalties. ${ }^{1}$ Shape anisotropy in magnetic micro- and nanodiscs supports several arrangements of magnetic spins with varied net magnetizations (Fig. 1a-c). The negligible net magnetic moment associated with a vortex state in magnetic microand nanodiscs minimizes magnetic dipole-dipole interactions between particles and is responsible for their enhanced colloidal stability ${ }^{2}$ compared to materials with similar magnetic volumes but uniform spin configurations. This colloidal stability motivated their previous biomedical applications; for example, the transition between vortex and in-plane magnetization states (Fig. 1d) in permalloy microdiscs has been leveraged to exert torque on cell membranes in the presence of 
weak magnetic fields. ${ }^{3}$ Microfabricated permalloy microdiscs, however, face challenges regarding their scalable synthesis and long-term chemical stability in physiological environments. ${ }^{3} \mathrm{We}$ hypothesized that colloidal synthesis of magnetic nanodiscs (MNDs) composed of stable magnetite would address these concerns, motivating the use of MNDs as transducers of signals in biological circuits.

Guided by magnetic modelling, we synthesized a broad palette of magnetite MNDs with diameters ranging from 98-226 nm and thicknesses between $24-37 \mathrm{~nm}$. Electron holography was then applied to visualize the alignment of magnetic spins in these MNDs, revealing magnetic vortex states. ${ }^{4}$ In weak, slowly varying magnetic fields, MNDs exerted tunable torques on the membranes of mechanosensitive cells, mediating remote control of biological signalling. Together, these findings indicate that tuning the vortex state in MNDs may be applied to future studies of mechanotransduction and remote torque-dependent switching of nanoscale processes.

\section{Model-driven synthesis and characterization of magnetite nanodises}

Tuning the dimensions of magnetite MNDs allowed for the identification of particles that support the magnetic vortex state, while boasting high induced moments in external MFs. Using The National Institute of Standards and Technology's Object Oriented MicroMagnetic Framework (OOMMF) ${ }^{5}$ we constructed a phase diagram showing the highest probability configurations of magnetic spins in magnetite MNDs as a function of their geometric aspect ratio (Fig. 1e). Guided by these simulations, we produced an array of MNDs with geometries that were predicted to exhibit the magnetic vortex state (markers in Fig. 1e).

The synthesis of anisotropic nanoparticles of the biochemically inert and stable mineral magnetite $\left(\mathrm{Fe}_{3} \mathrm{O}_{4}\right)$ is challenging due to its inverse spinel lattice with cubic symmetry, which favours isotropic growth (Fig. 2a). ${ }^{6}$ To address this challenge we have adapted a high-throughput protocol that relies on a solvothermal synthesis of hematite $\left(\alpha-\mathrm{Fe}_{2} \mathrm{O}_{3}\right)$ nanodiscs with a hexagonal lattice, followed by their reduction into magnetite MNDs with conserved dimensions. ${ }^{7}$ Briefly, $\mathrm{FeCl}_{3}$, sodium acetate, ethanol and water were heated in an autoclave reactor to generate hematite nanodiscs. After washing in deionized water and drying, the nanodiscs were resuspended in trioctylamine and oleic acid and heated in a hydrogen atmosphere to be converted to magnetite (Fig. 2a). 
Tuning the amount of water in the solution enabled aspect ratio tuning of the hematite nanodiscs. Water molecules allow for hydrogen bonding between the terminating oxygen atoms in the [0001] crystal plane of the hematite and the ligands (ethanol or acetate) present during synthesis, which inhibits the growth in the [0001] direction and results in a decrease in nanodisc diameter. ${ }^{8}$ Hematite nanodiscs with mean diameters of $244 \pm 37 \mathrm{~nm}, 181 \pm 29 \mathrm{~nm}, 166 \pm 28 \mathrm{~nm}, 159 \pm 26 \mathrm{~nm}$, and $103 \pm 24$ $\mathrm{nm}$ were produced in the presence of $6 \%, 7 \%, 8 \%, 9 \%$, and $10 \% \mathrm{v} / \mathrm{v}$ water in the reaction mixture, respectively (Fig. 2b-f). While the hexagonal shape was observed in nanodiscs prepared with 6$9 \%$ water (Fig. $2 \mathrm{~b}-\mathrm{e}$ ), the smallest particles prepared with $10 \%$ water displayed more isotropic shapes (Fig. 2f). Water content exceeding 10\% led to a further decrease in anisotropy, resulting in spheroidal hematite nanoparticles (Supplementary Fig. S1).

The reduction of hematite nanodiscs in a hydrogen atmosphere yielded complete conversion of non-magnetic hematite into magnetite while maintaining their geometry (Fig. 2g-n, Supplementary Fig. S2a). Mean diameters of magnetite nanodiscs were $226 \pm 37 \mathrm{~nm}, 181 \pm 17 \mathrm{~nm}, 170 \pm 22 \mathrm{~nm}$, $161 \pm 19 \mathrm{~nm}$ and $98 \pm 12 \mathrm{~nm}$ for $6 \%, 7 \%, 8 \%, 9 \%$, and $10 \% \mathrm{v} / \mathrm{v}$ water in the starting reaction mixture, respectively (Fig. 21). Scanning electron microscope images of the reduced nanodiscs indicated preservation of their shapes and dimensions (Fig. 2g-1), while X-ray diffraction spectra confirmed the transformation from hexagonal hematite into the magnetite inverse spinel phase (Fig. $2 \mathrm{~m}$, Supplementary Fig. S2b,c). ${ }^{9}$ This was consistent with the observation that the nanodisc saturation magnetization $\left(\mathrm{M}_{\mathrm{s}}\right)$ approached that of bulk magnetite $\left(115-120 \mathrm{emu} / \mathrm{g}_{\mathrm{Fe}}\right)(\mathrm{Fig} .2 \mathrm{n}){ }^{10}$

\section{Observation of the magnetic vortex state in magnetite nanodiscs}

Following synthesis and structural characterization, we sought to investigate the ground magnetization state of the magnetite MNDs. Micromagnetic simulations performed using OOMMF indicated that the magnetite MNDs with diameters of $226 \mathrm{~nm}$ and $181 \mathrm{~nm}$ should assume a vortex state (Fig. 3a,b) while the MNDs $98 \mathrm{~nm}$ in diameter should exhibit an in-plane spin configuration (Fig. 3c). To test these predictions against experimental data, we employed electron holography, which enables direct interrogation of the magnetization configuration in magnetic nanoparticles. ${ }^{11}$ While the vortex state in magnetic anisotropic particles has been extensively studied via physical modelling and magnetic force microscopy, ${ }^{12}$ the application of electron holography to characterize this state in magnetite nanoparticles has been limited, with no 
exploration of MNDs with varied dimensions and aspect ratios. ${ }^{13}$

In contrast to OOMMF simulations, electron holography revealed that the magnetic vortex state was not only present in magnetite MNDs with diameters of $\sim 226 \mathrm{~nm}$ and $\sim 181 \mathrm{~nm}$ but also in smaller $\sim 98 \mathrm{~nm}$ MNDs (Fig. 3d-f). This illustrated the limitations of the idealized assumptions used in the models. As anticipated for the vortex configuration, the particles had zero net magnetization with no measurable surrounding demagnetization field (Fig. 3g-i). The magnetization field in individual particles approached a maximum of $0.6 \mathrm{~T}$ locally (Fig. 3g,i), corresponding to $\mathrm{M}_{\mathrm{s}}=128 \mathrm{emu} / \mathrm{g}_{\mathrm{Fe}}$, consistent with our measurements (Fig. $2 \mathrm{n}$ ) and bulk values

reported for magnetite. ${ }^{10}$ In two stacked MNDs we observed vortices with opposite handedness (Fig. 3e, red and blue). The stacking and the differences in thickness between the MNDs resulted in overestimation of the magnetic field (up to $2 \mathrm{~T}$ ) (Fig. 3h) and $\mathrm{M}_{\mathrm{s}}$ compared to the values measured by ensemble magnetometry (Fig. 2n). The magnetization in clustered particles is further determined by the particle arrangement and its direction may partially align with the stack axis (Supplementary Fig. S3). Such stacking of MNDs occurs during the sample preparation for electron microscopy and is not anticipated in colloidal solutions, as indicated by dynamic light scattering (DLS) measurements (Supplementary Fig. S4). ${ }^{14}$

\section{Application of magnetic nanodiscs as transducers of nanoscale torques on cell membranes}

To render MNDs colloidally stable in physiological fluids and facilitate their attachment to cell membranes, these particles were functionalized with the amphiphilic ligand poly(maleic anhydride-alt-1-octadecene) (PMAO). ${ }^{15}$ The presence of the PMAO coating on the MNDs was confirmed by measuring the resulting negative zeta potential of $-44 \pm 3 \mathrm{mV}$, and colloidal stability was corroborated via DLS (Supplementary Fig. S4).

We hypothesized that the transition from a vortex to an in-plane magnetization state under applied magnetic field would allow MNDs decorating the cell membrane to exert torque mimicking biological mechanotransduction (Fig. 4a). The torque produced is proportional to the magnetic moment of a MND (which is proportional to the MND volume) and the strength of the applied magnetic field (MF) (Supplementary Information). To illustrate the effects of local MNDmediated torques on cell membranes, we incubated cultures of dorsal root ganglia explants (DRGs) with MNDs (Fig. 4b,c, Supplementary Fig. S5). DRGs contain sensory neurons expressing a wide 
range of mechanoreceptors. ${ }^{16,17}$ As mechanotransduction in sensory neurons involves changes in membrane potential and calcium-ion influx (Fig. 4a), the effects of magnetically mediated torques on the cells decorated with MNDs can be monitored via the fluorescence change of the $\mathrm{Ca}^{2+}$ indicator Fluo-4.

For the same MF conditions, larger MNDs are expected to exert greater torques on the cell membrane, and hence we examined the response of DRG neurons incubated with MNDs $226 \mathrm{~nm}$ or $98 \mathrm{~nm}$ in diameter. A low MND concentration of $60 \mu \mathrm{g} / \mathrm{ml}$ in Tyrode's solution was sufficient due to electrostatic attachment of MNDs to DRGs. Weak (26 mT), slowly varying (5 Hz) MFs were applied in $10 \mathrm{~s}$ epochs separated by $30 \mathrm{~s}$ rest periods via a custom-built apparatus compatible with simultaneous imaging (Supplementary Fig. S6). While the solution temperature remained within $0.5{ }^{\circ} \mathrm{C}$ (Fig. 4d), a rapid increase in relative Fluo-4 fluorescence normalized to the prestimulus average $\left(\Delta \mathrm{F} / \mathrm{F}_{0}\right)$ was observed in cells decorated with MNDs of either diameter (Fig. 4e-h, Supplementary Fig. 7a,b).

As predicted by torque calculations (Supplementary Information), $226 \mathrm{~nm}$ MNDs mediated a greater fluorescence increase in the examined cells than the $98 \mathrm{~nm}$ MNDs (Fig. 4f-h). Negligible responses to MF were found in cells not incubated with MNDs (Fig. 4h, Supplementary Fig. S7c,d). To further correlate the effects of MF stimulus to the torque-based mechanism, we have examined the responses of MND-decorated hippocampal neurons, which have limited mechanosensitivity. ${ }^{18}$ Negligible responses to MF were observed in hippocampal neurons in the presence of $98 \mathrm{~nm}$ MNDs (Fig. 4h, Supplementary Fig. S7e,f). In the presence of $226 \mathrm{~nm}$ MNDs, hippocampal neurons responded to MF at a significantly lower rate than the mechanosensitive DRG neurons. This minor, but observable response could likely be attributed in part to the changes in kinetics of ion channels expressed in hippocampal neurons as well as the direct effects on membrane permeability. ${ }^{18,19}$

To determine the MF magnitude and frequency thresholds for magnetomechanical stimulation, we performed $\mathrm{Ca}^{2+}$ imaging of DRGs incubated with MNDs of either $98 \mathrm{~nm}$ or $226 \mathrm{~nm}$ diameter subjected to MFs of amplitudes from 7-28 mT at either $1 \mathrm{~Hz}$ or $5 \mathrm{~Hz}$. We found that the highest frequencies and amplitudes resulted in the highest percentages of responding cells. The one exception to this trend was that $1 \mathrm{~Hz}$ MF stimulation outperformed $5 \mathrm{~Hz}$ stimulation at lower MF amplitudes (Fig. 5a,b, Supplementary Fig. S8), possibly because the response frequency of the 
nanodiscs is greater than $200 \mathrm{~ms}$ at lower MF amplitudes, because the torque driving their response is lower.

To further explore whether the neuronal activation caused by the MND torque is mediated by mechanosensitive ion channels, we specifically inhibited two major mechanoreceptors present in DRGs. PIEZO2, a recently described mechanosensitive channel, is abundant in DRG neurons and generates robust mechanosensitive cation currents. ${ }^{16}$ Another distinct mechanoreceptor in DRGs is the transient receptor potential vanilloid family member 4 (TRPV4). ${ }^{20}$ TRPV4 has been shown to be sufficient to impart sensitivity to $\mathrm{pN}$-range forces to non-mechanoreceptive cells. ${ }^{21}$

We first inhibited PIEZO2 by incubating DRGs with $1 \mu \mathrm{M}$ GsMTx4, the venom of the Chilean rose tarantula spider (Grammostola spatulata) that specifically blocks PIEZO channels. ${ }^{22}$ For selective blocking of TRPV4 we incubated DRGs with $1 \mu \mathrm{M}$ TRPV4 antagonist, HC-067047. ${ }^{23}$ MND-decorated DRGs, incubated with GsMTx4 (Fig. 5c, left panel and Supplementary Fig. S9a) and HC-067047 (Fig. 5c, right panel and Supplementary Fig. S9b) displayed similar behaviour when MF (5 Hz, $26 \mathrm{mT})$ was applied. The first $10 \mathrm{~s} \mathrm{MF}$ pulse produced a fluorescence change similar to trials without drugs, but the following two pulses produced negligible average fluorescence change (Fig. 5c, Supplementary Fig. S9a,b), indicating that individually inhibiting mechanosensitive channels significantly reduces the efficacy of magnetomechanical stimulation. The delay in the efficacy of the drugs may be due to the need for channel blockers to enter the pore of ion channels before they are inactivated. Similarly, sipatrine, an inhibitor of voltage-gated sodium channels and the mechanosensitive $\mathrm{K}^{+}$channels TREK-1 and TRAAK, ${ }^{24}$ decreased but did not completely eliminate the response to magnetomechanical stimulation (Supplementary Fig. $\mathrm{S} 10 \mathrm{a}$ ), indicating that the response is mediated by multiple classes of ion channels. In contrast, tetrodotoxin and palmitoleic acid, inhibitors of sodium channels and gap junctions in neurons and glia, respectively, failed to create any changes in the response to magnetomechanical stimulation, indicating that network effects are not responsible for a significant fraction of the response (Supplementary Fig. S10b-d).

Many types of mechanosensitive ion channels have been identified in DRGs. Moreover, certain voltage- or ligand-gated channels present in DRGs can also exhibit some mechanosensitivity and contribute to the response to magnetomechanical stimulation. ${ }^{25,26}$ For this reason, we used a nonmechanosensitive cell line, human embryonic kidney (HEK-293) cells, as a test bed for 
magnetomechanical stimulation (Fig. 5d). We also introduced mechanosensitivity to HEK-293 cells using transfection with a pLenti-CamKII $\alpha:$ TRPV4-p2A-mCherry plasmid developed inhouse (Supplementary Fig. S11a). ${ }^{27}$ Naive HEK-293 cells are inactive during application of magnetomechanical stimulation (Fig. 5e, left panel), but TRPV4 ${ }^{+}$HEK-293 cells displayed mechanosensitivity (Fig. 5e, middle panel, Supplementary Fig. S11b). This mechanosensitivity is then inhibited with the TRPV4 antagonist HC-067047 (Fig. 5e, right panel, Supplementary Fig. S11c), confirming that magnetomechanical stimulation with MNDs could be mediated by individual classes of mechanosensitive ion channels.

While the biocompatibility of isotropic magnetite nanoparticles with dimensions below $100 \mathrm{~nm}$ is well documented ${ }^{28}$ the effects of MNDs on cell viability have not yet been explored. Incubating DRGs with MNDs for 8 days in vitro did not yield significant changes in cell viability (Supplementary Fig. S12a). In addition, we investigated whether the MF-mediated torques transduced by MNDs to cell membranes could potentially compromise the membrane integrity. In DRG cultures decorated with $226 \mathrm{~nm}$ MNDs, $26 \mathrm{mT}, 5 \mathrm{~Hz}$ MFs did not yield measurable changes in cell viability (Supplementary Fig. S12b-d). This observation indicates that the modest responses to MFs in hippocampal neurons decorated with $226 \mathrm{~nm}$ MNDs were likely mediated by the effects of the torque on ion channel gating rather than on membrane permeability.

We hypothesized that switching between the vortex and in-plane magnetization states in anisotropic nanomaterials may permit their use as transducers of torque at the nanoscale. Guided by the predictions of the magnetization state in disc-shaped magnetite particles, we have synthesized an array of MNDs with diameters 98-226 nm and thicknesses 24-37 nm. Electron holography measurements revealed the magnetic vortex ground magnetization state in MNDs with diameters as low as $98 \mathrm{~nm}$. To evaluate the ability of the MNDs to transduce local torques, we applied them to drive the activity of mechanoreceptive sensory neurons in the presence of weak, slowly varying MFs. The extent of the neuronal response was correlated with the MND volume and the MF strength, consistent with a torque-based mechanism. Furthermore, experiments in multiple cell types conducted in the presence of two MND sizes and varying MF parameters indicated that MND-mediated torques drive cellular signalling by activating ion channels involved in mechanotransduction rather than by directly changing the membrane permeability. Our findings indicate that tuning the vortex state in MNDs may be applied to future studies of force transduction at the nanoscale, including the investigation of biological mechanoreception. 


\section{Acknowledgments}

We thank Feng Zhang (MIT) for the gift of the HEK-293FT cell line. The authors are also grateful to Michael G. Christiansen, Siyuan Rao, Seongjun Park, and Gabriela Romero for their technical advice on the experiments and apparatus. This study was supported in part by the Defense Advanced Research Project Agency (DARPA) through the ElectRx (HR0011-15-C-0155) program, National Institute of Mental Health through the BRAIN Initiative (1R01MH111872-01), and made use of the facilities available through the National Science Foundation (NSF) Center for Materials Science and Engineering (DMR-1419807). This research was conducted with government support awarded by the DoD, the Air Force Office of Scientific Research, and the National Defense Science and Engineering Graduate (NDSEG) Fellowship, 32 CFR 168a, which funded A.W.S. A.C. acknowledges support from the Spanish Ministry of Economy, Industry and Competitiveness under the Maria de Maeztu Units of Excellence Programme MDM-2016-0618.

\section{Author contributions}

D.G., A.W.S., and P.A. designed the experiments and wrote the manuscript. D.G., A.W.S., A.C., P.D.R., A.S., D.R., P.-H.C. and F.G. performed the experiments. G.V. and E.C. contributed to the development of the OOMMF models.

\section{Competing financial interest statement}

P.A., A.W.S., D.G., and P.D.R. are authors of a patent application based on this work.

\section{Data availability}

The data that support the findings of this study are available within the manuscript and supplementary information. Raw data associated with main text Figures 1d-e, 21-n, 3, 4d-h, 5a-c,e and Supplementary Figures 3, 4, 7, 8, 9, 10, 11 and 12a,b are available from the corresponding author upon request. Custom analysis scripts are available from the corresponding author upon request. 


\section{References}

1. Parreiras SO, Martins MD. Effect of Planar Anisotropy in Vortex Configuration of Microscale Disks. Physics Procedia 2015, 75: 1142-1149.

2. Lisjak D, Mertelj A. Anisotropic magnetic nanoparticles: A review of their properties, syntheses and potential applications. Progress in Materials Science 2018, 95: 286-328.

3. Kim D-H, Rozhkova EA, Ulasov IV, Bader SD, Rajh T, Lesniak MS, et al. Biofunctionalized magnetic-vortex microdiscs for targeted cancer-cell destruction. Nature Materials 2009, 9: 165.

4. Ammar M, LoBue M, Snoeck E, Hÿtch M, Champion Y, Barrué R, et al. A quantitative analysis of magnetic vortices in Permalloy nanoparticles characterized by electron holography. Journal of Magnetism and Magnetic Materials 2008, 320(20): e716-e719.

5. Donahue MJ. OOMMF user's guide [microform] / M.J. Donahue, D. G. Porter. U.S. Dept. of Commerce, Technology Administration, National Institute of Standards and Technology: Gaithersburg, MD, 1999.

6. Mann S, Sparks NHC, Blakemore RP. Structure, Morphology and Crystal Growth of Anisotropic Magnetite Crystals in Magnetotactic Bacteria. Proceedings of the Royal Society of London Series B, Biological Sciences 1987, 231(1265): 477-487.

7. Zhu M, Wang Y, Meng D, Qin X, Diao G. Hydrothermal Synthesis of Hematite Nanoparticles and Their Electrochemical Properties. The Journal of Physical Chemistry C 2012, 116(30): 16276-16285.

8. Chen L, Yang X, Chen J, Liu J, Wu H, Zhan H, et al. Continuous Shape- and SpectroscopyTuning of Hematite Nanocrystals. Inorganic Chemistry 2010, 49(18): 8411-8420.

9. Chen R, Christiansen MG, Anikeeva P. Maximizing Hysteretic Losses in Magnetic Ferrite Nanoparticles via Model-Driven Synthesis and Materials Optimization. ACS Nano 2013, 7(10): 8990-9000.

10. Kemp SJ, Ferguson RM, Khandhar AP, Krishnan KM. Monodisperse magnetite nanoparticles with nearly ideal saturation magnetization. RSC Advances 2016, 6(81): $77452-77464$.

11. E. DBR, Takeshi K, Alexander W, L. TS, J. HM, Etienne S, et al. Off-axis electron holography of magnetic nanowires and chains, rings, and planar arrays of magnetic nanoparticles. Microscopy Research and Technique 2004, 64(5-6): 390-402.

12. Yang Y, Liu X-L, Yi J-b, Yang Y, Fan H-M, Ding J. Stable vortex magnetite nanorings colloid: Micromagnetic simulation and experimental demonstration. Journal of Applied Physics 2012, 111(4): 044303.

13. Yong Y, Xiaoli L, Yunbo L, Seng HT, Xianhui X, Weixing X, et al. Orientation Mediated Enhancement on Magnetic Hyperthermia of Fe3O4 Nanodisc. Advanced Functional Materials 2015, 25(5): 812-820.

14. Fissan H, Ristig S, Kaminski H, Asbach C, Epple M. Comparison of different characterization methods for nanoparticle dispersions before and after aerosolization. Analytical Methods 2014, 6(18): 7324-7334.

15. Pichaandi J, Abel KA, Johnson NJJ, van Veggel FCJM. Long-Term Colloidal Stability and Photoluminescence Retention of Lead-Based Quantum Dots in Saline Buffers and Biological Media through Surface Modification. Chemistry of Materials 2013, 25(10): 2035-2044. 
16. Coste B, Mathur J, Schmidt M, Earley TJ, Ranade S, Petrus MJ, et al. Piezo1 and Piezo2 Are Essential Components of Distinct Mechanically Activated Cation Channels. Science 2010, 330(6000): 55-60.

17. Ranade SS, Syeda R, Patapoutian A. Mechanically Activated Ion Channels. Neuron 2015, 87(6): 1162-1179.

18. Paoletti P, Ascher P. Mechanosensitivity of NMDA receptors in cultured mouse central neurons. Neuron 1994, 13(3): 645-655.

19. Tay A, Kunze A, Murray C, Di Carlo D. Induction of Calcium Influx in Cortical Neural Networks by Nanomagnetic Forces. ACS Nano 2016, 10(2): 2331-2341.

20. Cao D-S, Yu S-Q, Premkumar LS. Modulation of transient receptor potential vanilloid 4mediated membrane currents and synaptic transmission by protein kinase C. Molecular Pain 2009, 5: 5-5.

21. Matthews BD, Thodeti CK, Tytell JD, Mammoto A, Overby DR, Ingber DE. Ultra-rapid activation of TRPV4 ion channels by mechanical forces applied to cell surface beta1 integrins. Integr Biol (Camb) 2010, 2(9): 435-442.

22. Bae C, Sachs F, Gottlieb PA. The mechanosensitive ion channel Piezo1 is inhibited by the peptide GsMTx4. Biochemistry 2011, 50(29): 6295-6300.

23. Garcia-Elias A, Mrkonjic S, Pardo-Pastor C, Inada H, Hellmich UA, Rubio-Moscardo F, et al. Phosphatidylinositol-4,5-biphosphate-dependent rearrangement of TRPV4 cytosolic tails enables channel activation by physiological stimuli. Proceedings of the National Academy of Sciences of the United States of America 2013, 110(23): 9553-9558.

24. Meadows HJ, Chapman CG, Duckworth DM, Kelsell RE, Murdock PR, Nasir S, et al. The neuroprotective agent sipatrigine (BW619C89) potently inhibits the human tandem poredomain $\mathrm{K}(+)$ channels TREK-1 and TRAAK. Brain research 2001, 892(1): 94-101.

25. Gu CX, Juranka PF, Morris CE. Stretch-activation and stretch-inactivation of Shaker-IR, a voltage-gated K+ channel. Biophysical journal 2001, 80(6): 2678-2693.

26. Mienville J, Barker JL, Lange GD. Mechanosensitive properties of BK channels from embryonic rat neuroepithelium. The Journal of membrane biology 1996, 153(3): 211-216.

27. Avci-Adali M, Behring A, Keller T, Krajewski S, Schlensak C, Wendel HP. Optimized conditions for successful transfection of human endothelial cells with in vitro synthesized and modified mRNA for induction of protein expression. J Biol Eng 2014, 8(1): 8-8.

28. Chen R, Romero G, Christiansen MG, Mohr A, Anikeeva P. Wireless magnetothermal deep brain stimulation. Science 2015, 347(6229): 1477-1480.

29. Jia H, Rochefort NL, Chen X, Konnerth A. In vivo two-photon imaging of sensory-evoked dendritic calcium signals in cortical neurons. Nature Protocols 2010, 6: 28.

30. Momma K, Izumi F. VESTA 3 for three-dimensional visualization of crystal, volumetric and morphology data. Journal of Applied Crystallography 2011, 44(6): 1272-1276. 
a

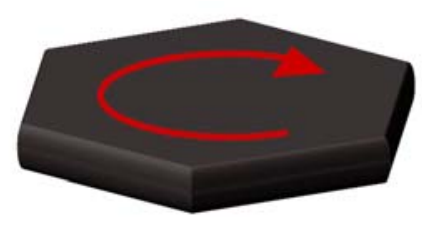

d

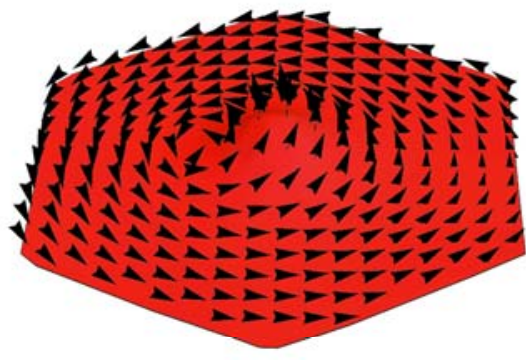

b

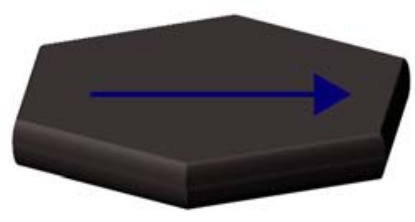

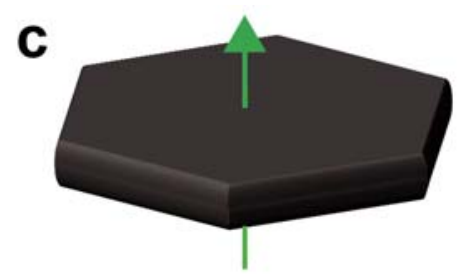
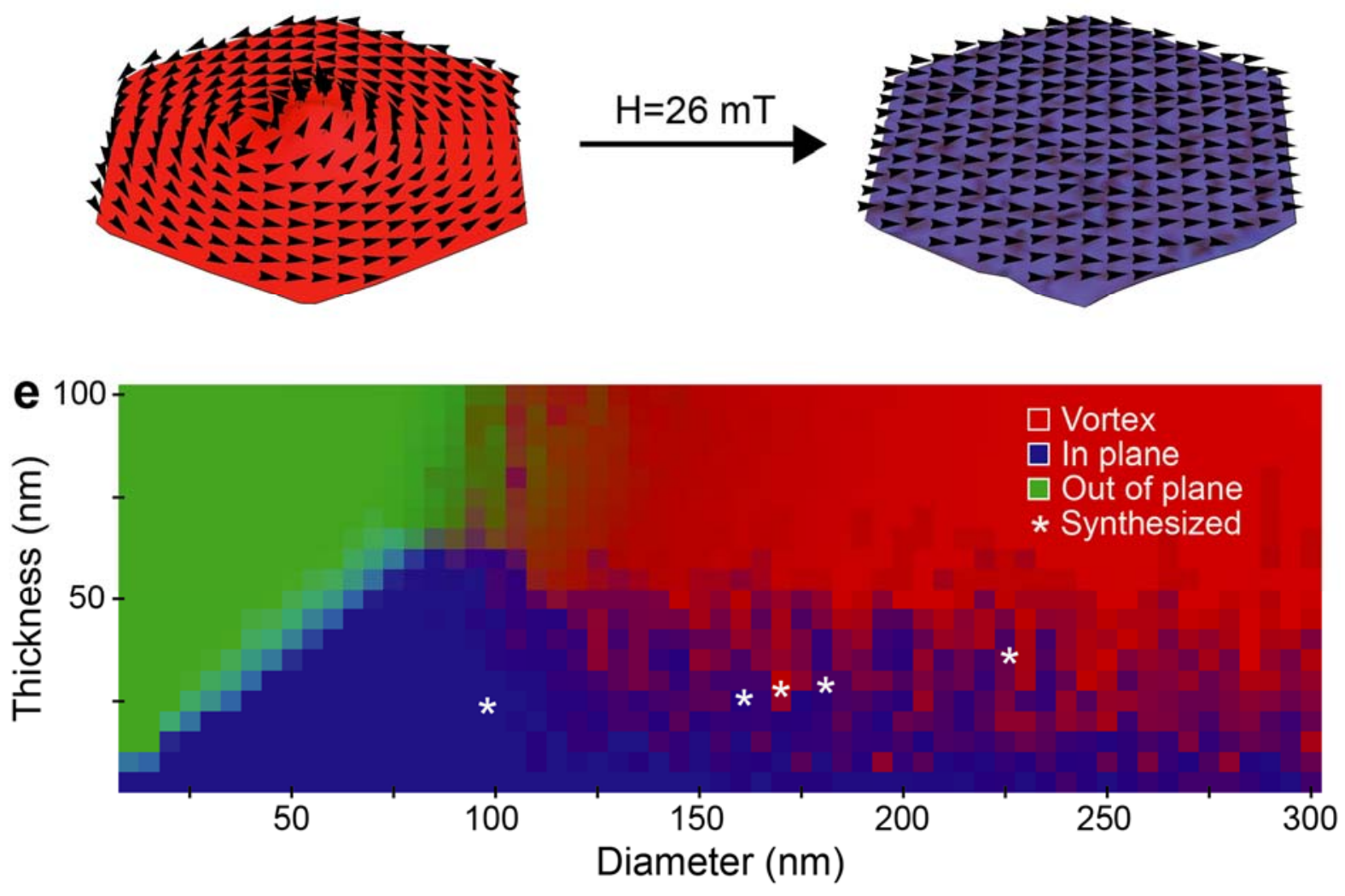

Fig. 1: The magnetic vortex phase in magnetite nanodiscs.

a-c, Schematic representation of the three possible configurations of magnetic spins in magnetite nanodiscs; a, vortex, $\mathbf{b}$, in-plane and $\mathbf{c}$, out-of-plane. d, MND that supports the magnetic vortex state (left) in the absence of applied magnetic field can transition to being magnetized in-plane (right) upon the application of a weak magnetic field. e, MNDs with a range of thicknesses and diameters were simulated using OOMMF, and their lowest energy configuration was plotted to create a magnetic domain state phase diagram. For points with an intermediate colour, either the equilibrium phase is a hybrid of the three canonical phases or two distinct equilibrium phases can result from different initial conditions in the simulation. White star markers on the phase diagram represent five MND compositions synthetized and used for experiments in this study. 

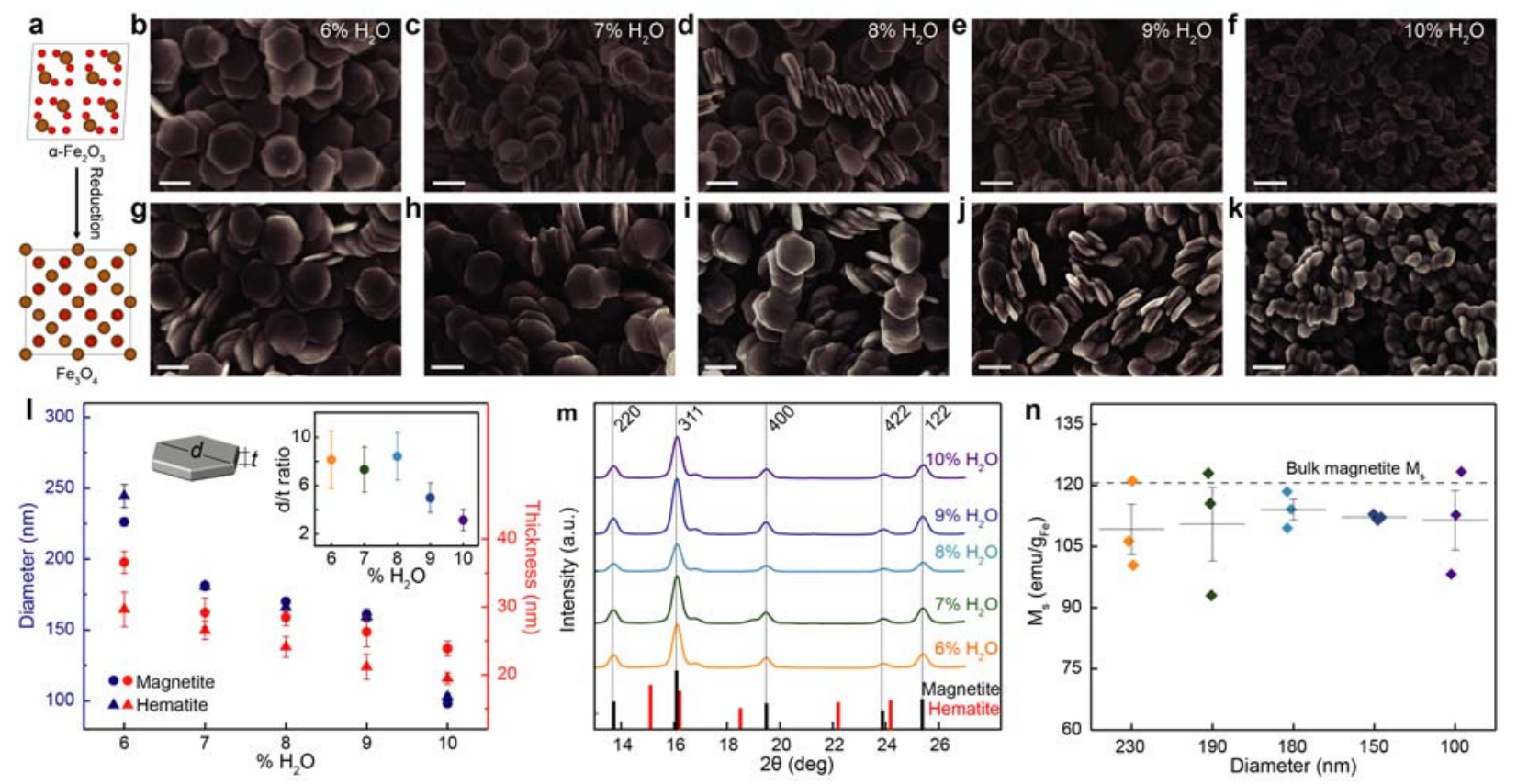

Fig. 2: Synthesis and characterization of magnetite nanodiscs.

a, Crystal units of non-magnetic hematite phase converted to magnetic magnetite after reduction. Nanodiscs synthetized in two-step solvothermal reaction with $6 \% \mathrm{H}_{2} \mathrm{O}(\mathbf{b}, \mathbf{g}), 7 \% \mathrm{H}_{2} \mathrm{O}(\mathbf{c}, \mathbf{h}), 8 \%$ $\mathrm{H}_{2} \mathrm{O}(\mathbf{d}, \mathbf{i}), 9 \% \mathrm{H}_{2} \mathrm{O}(\mathbf{e}, \mathbf{j})$ and $10 \% \mathrm{H}_{2} \mathrm{O}$. (f, k). b-f are hematite structures $\left(\alpha-\mathrm{Fe}_{2} \mathrm{O}_{3}\right)$ resulting from the first step of the synthesis. $\mathbf{g}-\mathbf{k}$ are magnetite structures $\left(\mathrm{Fe}_{3} \mathrm{O}_{4}\right)$ derived from the reduction of hematite discs. Scale bars $=200 \mathrm{~nm}$. 1, Diameter and thickness of nanodiscs before and after reduction as a function of the percentage of $\mathrm{H}_{2} \mathrm{O}$ in the synthesis (inset: calculated $\mathrm{d} / \mathrm{t}$ ratio). $\mathbf{m}$, XRD spectra for MNDs after reduction (hematite reference in red, magnetite reference in black). $\mathbf{n}$, Saturation magnetization for MNDs and reference to saturation magnetization of bulk magnetite shown (dashed line). ${ }^{14}$ 

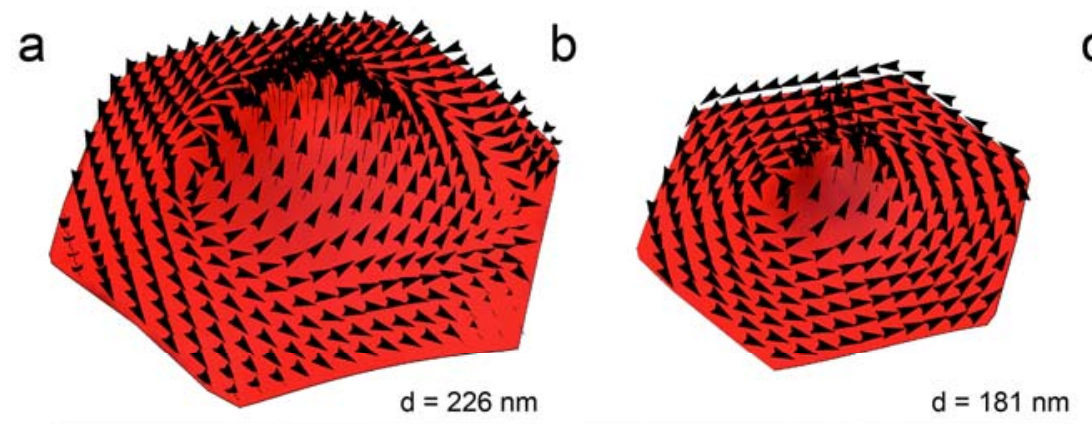

C

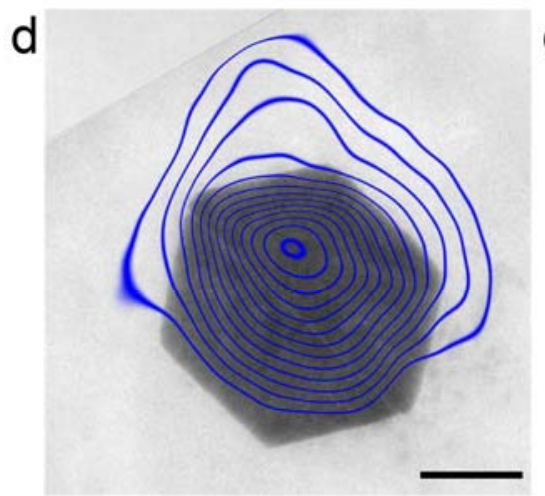

e

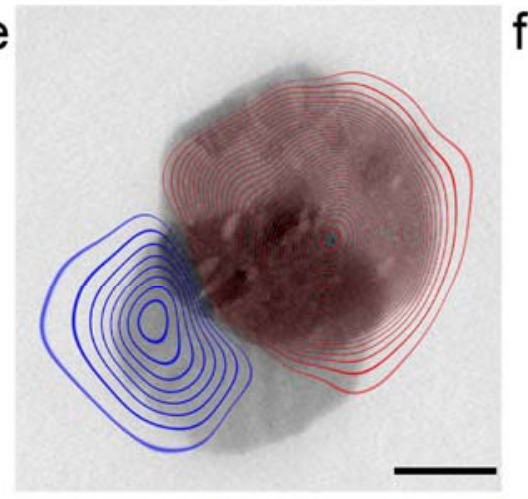

f
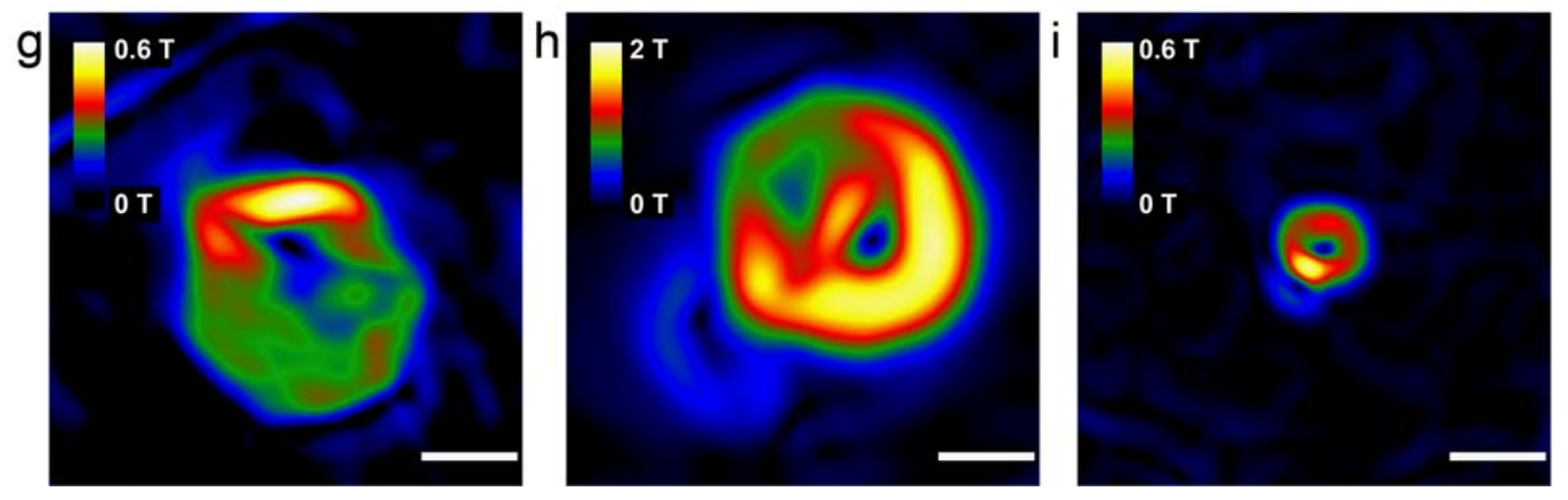

Fig. 3: Simulations and experimental study of ground vortex state in magnetite nanodiscs.

a-c, Ground state magnetization configuration resulting from micromagnetic simulation of MNDs $226 \mathrm{~nm}, 181 \mathrm{~nm}$, or $98 \mathrm{~nm}$ in diameter, respectively. d-i, Electron holography on MNDs: magnetic field lines overlapping the magnetic nanodiscs $226 \mathrm{~nm}, 181 \mathrm{~nm}$ or $98 \mathrm{~nm}$ in diameter, respectively. g-i, Corresponding magnetization of upper images. Scale bars $=100 \mathrm{~nm}$. 


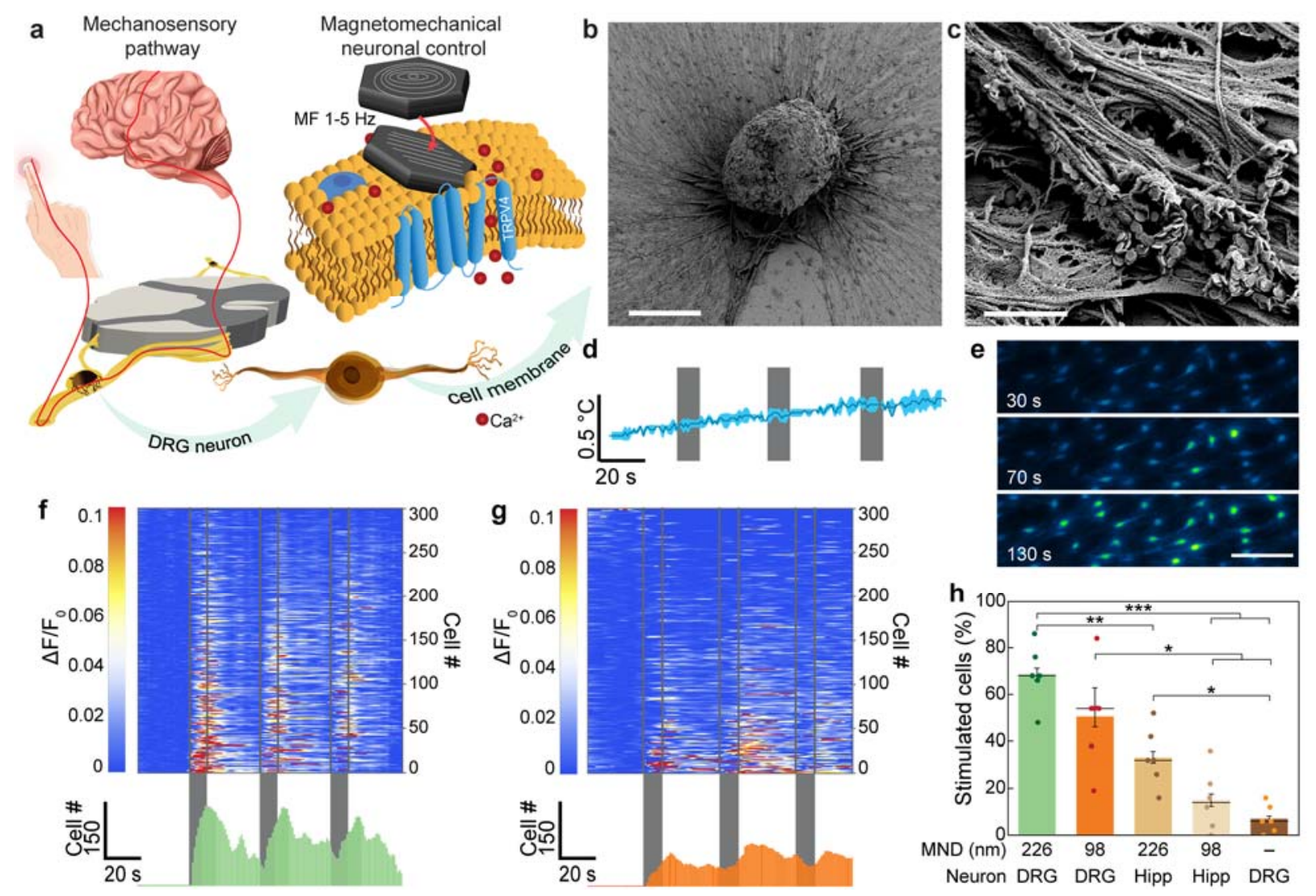

Fig. 4: Magnetomechanical stimulation of MND-decorated DRGs allows for remote activation of $\mathrm{Ca}^{2+}$ influx.

a, DRGs relay sensory information, including mechanosensory information, to the spinal cord. DRG explants incubated with MNDs can be stimulated when slowly varying MFs cause MNDs to transition from vortex to in-plane magnetization, producing forces on mechanosensitive ion channels and resulting in $\mathrm{Ca}^{2+}$ influx. $\mathbf{b}$, Scanning electron microscope image of DRG explant culture incubated with MNDs. Scale bar $=200 \mu \mathrm{m}$. $\mathbf{c}$, Detail of a region of the DRG. Individual MNDs are visible on the surface. Scale bar $=2 \mu \mathrm{m}$. d, Bath temperature monitored during a magnetomechanical stimulation experiment. Shaded area represents standard error. e, False colour stills from a representative video of DRG explant culture loaded with the $\mathrm{Ca}^{2+}$ indicator Fluo-4 and subject to magnetomechanical stimulation. Scale bar $=50 \mu \mathrm{m} .5 \mathrm{~Hz}, 26 \mathrm{mT}$ MF stimulation of DRGs decorated with MNDs $226 \mathrm{~nm}$ (f) and $98 \mathrm{~nm}$ (g) in diameter. Heat maps (top) represent the fluorescence change of individual cells during the experiment. On the same time axis are plotted the total number of responding cells at any time (bottom). $\mathbf{h}$, Comparison of the efficacy of magnetomechanical stimulation for $226 \mathrm{~nm}$ and $98 \mathrm{~nm}$ diameter MNDs on DRGs. A two-way 
ANOVA was conducted on the influence of culture type (DRG or hippocampal neurons) and MND type (226 nm, $98 \mathrm{~nm}$, and none) on the percentage of stimulated cells in calcium imaging. The main effect for MND type yielded an $F$ ratio of $F_{2,30}=38, p=5.6 \times 10^{-9}$. The main effect for culture type yielded an $\mathrm{F}$ ratio of $F_{1,30}=33, \mathrm{p}=2.8 \times 10^{-6}$. The interaction was significant $F_{2,30}=6.4, \mathrm{p}=$ $4.9 \times 10^{-3}$. In all experiments, MF is applied in 3 sequences of $10 \mathrm{~s}$ as shown in shaded grey regions. The cell number per condition is 300 , and the number of experiments per condition is $n=6$. All $p$ values from the Tukey-Kramer post-hoc test are listed in Supplementary Table 1. ${ }^{*} p \leq 0.05$, $* * \mathrm{p} \leq 0.01, * * * \mathrm{p} \leq 0.001$. 
a
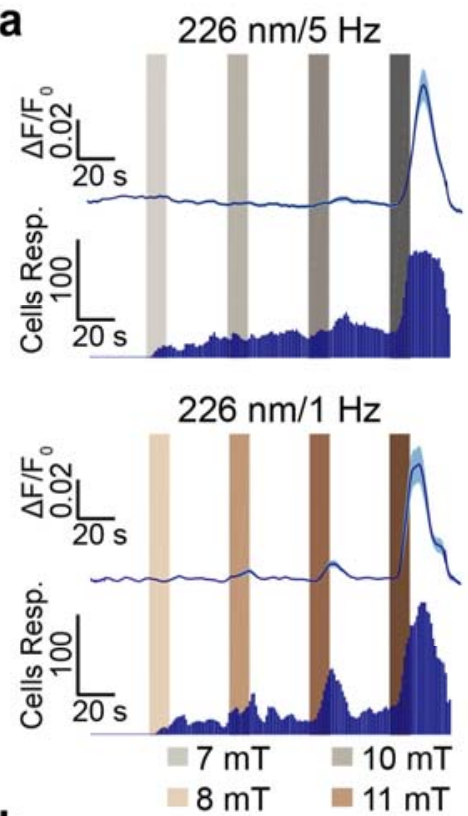

$98 \mathrm{~nm} / 5 \mathrm{~Hz}$

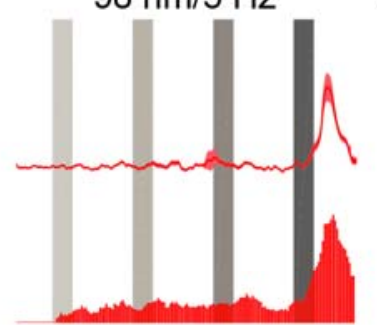

$98 \mathrm{~nm} / 1 \mathrm{~Hz}$

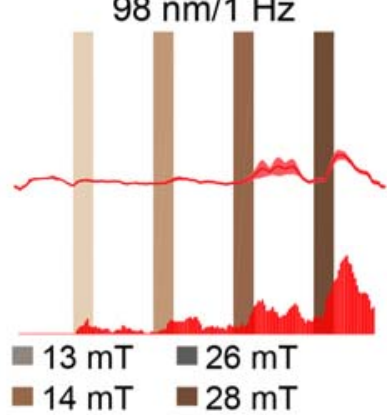

b

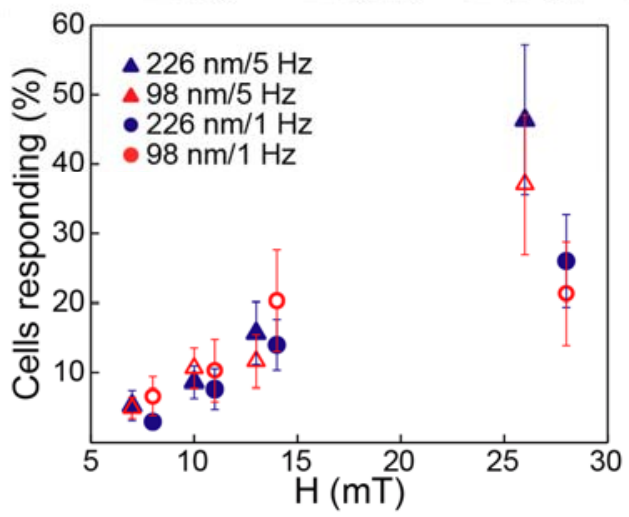

e
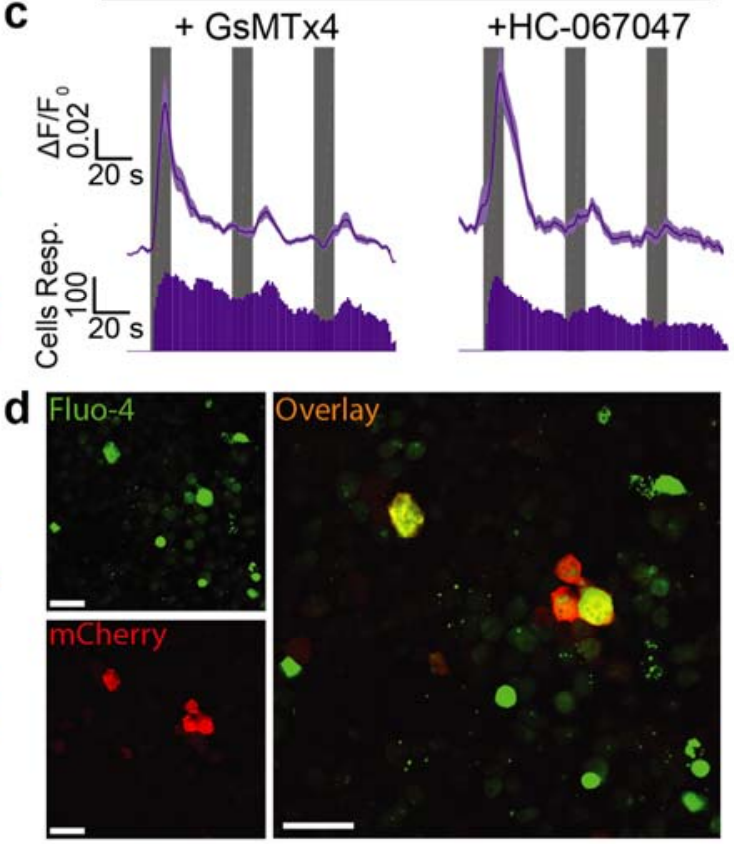

HEK

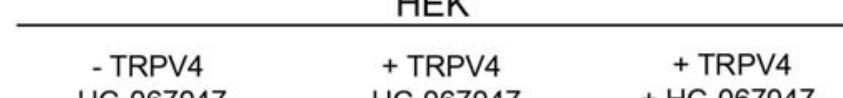
- HC-067047

- HC-067047

$+\mathrm{HC}-067047$

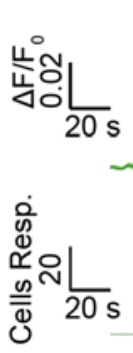

\section{$-\mathrm{HC}-067047$}
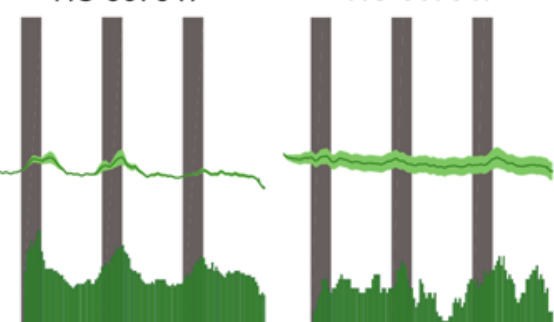

Fig. 5: Tuning parameters for magnetomechanical stimulation and demonstration of magnetomechanical stimulation in HEK-293 cells transfected with TRPV4.

a, Fluorescence traces resulting from stimulation of DRGs incubated with $226 \mathrm{~nm}$ diameter MNDs (left) and $98 \mathrm{~nm}$ diameter MNDs with MF frequencies of $5 \mathrm{~Hz}$ (top) and $1 \mathrm{~Hz}$ (bottom). MF amplitude is sequentially increased from $7 \mathrm{mT}$ to $28 \mathrm{mT}$ (marked by the shaded regions). MF is applied in 4 pulses of $10 \mathrm{~s}$ with $30 \mathrm{~s}$ wait times between pulses. b, Summary of cell response rate for conditions permuted in (a). Error bars represent standard error of the mean. c, DRGs incubated with $1 \mu \mathrm{M}$ piezo inhibitor GsMTx4 (left) and with TRPV4 antagonist HC-067047 (right) both show a decrease in activity after the first stimulation sequence. d, Confocal images of HEK-293 cells loaded with the calcium indicator Fluo-4 and transfected with the mechanosensitive TRPV4 channel labelled with mCherry. Scale bars $=100 \mu \mathrm{m}$. e, The response of unmodified HEK-293 cells decorated with MNDs to MF application (left), the response of HEK-293 cells expressing 
TRPV4 decorated with MNDs to MF application (middle), and the blocked response of HEK-293 cells decorated with MNDs, expressing TRPV4, and incubated $1 \mu \mathrm{M}$ TRPV4 antagonist, HC067047 (right). In experiments shown in (c) and (e), a $5 \mathrm{~Hz}, 26 \mathrm{mT} \mathrm{MF}$ is applied in 3 sequences of $10 \mathrm{~s}$ as shown in the shaded grey regions. The number of cells per condition is 300 . Standard error is represented by shaded area in fluorescence traces. 


\section{Methods}

Hematite and magnetite nanodisc synthesis. Magnetite nanodiscs were produced based on a two-step process by reducing hematite nanodiscs to magnetite nanodiscs. ${ }^{13}$ Hematite nanodiscs were synthetized by mixing $10 \mathrm{~mL}$ of absolute ethanol (VWR) and $0.8 \mathrm{~g}$ of anhydrous sodium acetate (Sigma-Aldrich) in glass beaker. Size of final magnetite phase was tuned by varying the $\mathrm{H}_{2} \mathrm{O}$ amount; $226 \mathrm{~nm}$ diameter $=0.6 \mathrm{~mL} \mathrm{H} \mathrm{H}_{2} \mathrm{O}, 181 \mathrm{~nm}$ diameter $=0.7 \mathrm{~mL} \mathrm{H} \mathrm{H}_{2} \mathrm{O}, 170 \mathrm{~nm}$ diameter $=0.8 \mathrm{~mL}, 161 \mathrm{~nm}$ diameter $=0.9 \mathrm{~mL} \mathrm{H}_{2} \mathrm{O}$ and $98 \mathrm{~nm}$ diameter $=1 \mathrm{~mL} \mathrm{H} \mathrm{H}_{2} \mathrm{O}$. The reaction mixture was magnetically stirred until homogenized. Then $0.273 \mathrm{~g} \mathrm{FeCl3} \cdot 6 \mathrm{H}_{2} \mathrm{O}$ (Fluka) was added and stirred. The homogenized mixture was moved to a Teflon-lined steel vessel, sealed, and heated to

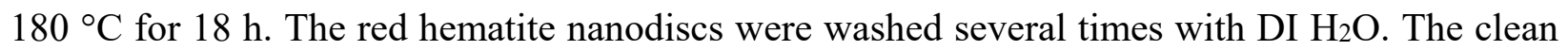
particles were then dispersed in ethanol and dried in a vacuum desiccator. In all the experiments, ultrapure (UP) water used was produced with a Milli-Q ${ }^{\circledR}$ UV water purification system (Millipore).

Hematite nanodiscs were reduced by adding $20 \mathrm{~mL}$ trioctylamine (Sigma-Aldrich) and $1 \mathrm{~g}$ oleic acid (Alfa Aesar). The mixture was placed in a three-neck flask connected to a Schlenk line and heated at $20^{\circ} \mathrm{C} / \mathrm{min}$ to reflux $\left(370^{\circ} \mathrm{C}\right)$ for $25 \mathrm{~min}$ in an atmosphere of $\mathrm{H}_{2}(5 \%)$ and $\mathrm{N}_{2}(95 \%)$. The mixture turns a metallic silver-black colour. Once cooled, the mixture was washed several times with hexane. The clean magnetite particles were then dispersed in chloroform (SigmaAldrich) and stored in a glass vial.

Water transfer. MNDs are coated in hydrophobic oleic acid following synthesis so they are functionalized with biocompatible poly(maleic anhydride-alt-1-octadecene) (PMAO) (average $\mathrm{M}_{\mathrm{n}}$ 30,000-50,000, Aldrich). First, a $10 \mathrm{mg} / \mathrm{mL}$ solution of PMAO in chloroform is prepared. Then this solution is added to dried nanodiscs $(1 \mathrm{mg} / \mathrm{mL}$, iron basis). This mixture is then sonicated for one minute and allowed to dry in a vacuum desiccator. When completely dry, TBE buffer (SigmaAldrich) was added and the nanodiscs were resuspended via sonication at $80^{\circ} \mathrm{C}$ for 2 hours. Then the particles were washed with $\mathrm{UP}_{2} \mathrm{O}$ and stored in $\mathrm{UP}_{2} \mathrm{O}$ in a glass vial.

Structural and magnetic characterization. Scanning electron microscopy of hematite and magnetite was performed with a Zeiss Merlin SEM. Transmission electron microscopy and singleparticle electron diffraction was performed using an FEI Tecnai G2 Spirit TWIN TEM. Powder $\mathrm{X}$-ray diffraction patterns of as-synthesized hematite and magnetite were collected on a three- 
circle diffractometer coupled to a Bruker-AXS Smart Apex charged-coupled-device (CCD) detector with graphite-monochromated Mo K $\alpha$ radiation $(\lambda=0.71073 \AA$ ) at $300 \mathrm{~K}$. Data were processed with the PANalytical HighScore Plus software. Room-temperature hysteresis curves were generated on a vibrating sample magnetometer (VSM, Digital Measurement Systems Model 880A). An Agilent 5100 Inductively Coupled Plasma-Optical Emission Spectrometer (ICP-OES) was used to quantify the elemental concentration for calculation of saturation magnetization $\left(\mathrm{M}_{\mathrm{s}}\right)$. For ICP-OES analysis, magnetite nanodiscs were digested in $37 \% \mathrm{v} / \mathrm{v} \mathrm{HCl}$ (Sigma-Aldrich) overnight at $60{ }^{\circ} \mathrm{C}$ and diluted in $2 \mathrm{wt} \% \mathrm{HNO}_{3}$ (Sigma-Aldrich).

Electron holography imaging and image processing. Electron holography measurements were performed on Titan 60-300 TEM (FEI Co., Netherlands) at $300 \mathrm{kV}$ accelerating voltage in an aberration corrected Lorentz mode. The microscope was equipped with an electron bi-prism at the position of the $\mathrm{SA}$ aperture. The bi-prism bias was at about $+250 \mathrm{~V}$, resulting in interference fringes with a period of about $1.3 \mathrm{~nm}$. In order to extract a magnetic contribution to the phase shift, the samples were ex-situ flipped and re-measured from the other side. Reconstructed phase images were post-processed in order to account for mirror symmetry, rotation and distortions caused by the corrector. The magnetic contribution to the phase shift was obtained as a difference of the images from two sides. The remnant magnetization was calculated from projected field values assuming average platelet thickness (measured independently).

OOMMF simulations. The National Institute of Standards and Technology's Object Oriented MicroMagnetic Framework (OOMMF) ${ }^{5}$ was used to simulate MNDs. A mask was used to define the hexagonal shape of a MND, and the MND was subdivided into $125 \mathrm{~nm}^{3}$ elements, each with a random initial magnetization direction. OOMMF then implements an energy minimization algorithm to find the lowest-energy configuration of element magnetizations (each element is treated as uniformly magnetized). The expected equilibrium domain states were in-plane, out-ofplane, and vortex, though sometimes intermediate phases were observed. An automated phase classification scheme was developed, in which the net in-plane and out-of-plane magnetization character of each simulated MND was calculated and converted to an RGB colour value. This colour value was plotted for MNDs with a range of dimensions and the result was a phase diagram indicating, for a given MND size, what the default magnetic domain state is.

Magnetic field system. A $10 \mathrm{lb}$ spool of 16 AWG magnet wire was purchased from MWS Wire 
Industries. The centre of this spool was removed to create a large-bore solenoid. A commercial magnetic field (MF) probe (Lake Shore Cryotronics) was used to measure the field/current relationship for the solenoid, and during experiments the current was measured via the voltage across a $0.5 \mathrm{ohm}$ shunt resistor connected in series with the solenoid. The measured current and the established field/current relationship was used to infer the MF amplitude. The solenoid/resistor circuit was driven by a Crown DC-300 power amplifier with a 3 VP-p, $5 \mathrm{~Hz}$ input signal from a function generator.

In vitro studies. All the experimental procedures involving primary cultures were approved by the MIT Committee on Animal Care.

Dorsal root ganglion (DRG) culture. $12 \mathrm{~mm}$ round glass cover slips were coated with $70 \mathrm{uL}$ Matrigel ${ }^{\circledR}$ solution (Corning ${ }^{\circledR}$ ) (Matrigel ${ }^{\circledR}$ diluted 30x in Neurobasal ${ }^{\mathrm{TM}}$ medium with serum-free B2 $7^{\mathrm{TM}}$ Supplement (both Gibco, ThermoFisher)) overnight prior to DRG seeding. Whole DRGs from P0 Sprague-Dawley rat pups were seeded individually on cover slips in a 24 well plate with $1 \mathrm{~mL}$ Neurobasal ${ }^{\mathrm{TM}}$ medium with serum-free $\mathrm{B} 27^{\mathrm{TM}}$ supplement per well and kept in incubator at $37^{\circ} \mathrm{C}$ and $5 \% \mathrm{CO}_{2}$.

Hippocampal neuron culture. Hippocampi were extracted from pups of Sprague-Dawley rats at post-natal day 0 in cold dissection solution $\left(160 \mathrm{mM} \mathrm{NaCl}, 5 \mathrm{mM} \mathrm{KCl}, 1 \mathrm{mM} \mathrm{MgSO}_{4}, 4 \mathrm{mM}\right.$ $\mathrm{CaCl}_{2}, 5 \mathrm{mM}$ HEPES, $5.5 \mathrm{mM}$ Glucose, adjusted to $\mathrm{pH} 7.4$ by $\mathrm{NaOH}$ ). To dissociate hippocampal neurons, extracted hippocampi were transferred into pre-warmed digestion solution $\left(1 \mathrm{mM} \mathrm{CaCl}_{2}\right.$, $1.5 \mathrm{mM} \mathrm{NaOH}, 0.5 \mathrm{mM}$ EDTA, $1 \mathrm{mM}$ L-cysteine, and 10 units $/ \mathrm{ml}$ papain (Worthington Biochemical) in dissection solution) and incubated at $37{ }^{\circ} \mathrm{C}$ for $25 \mathrm{~min}$. Digestion was stopped by incubating in inhibition solutions ( $0.25 \%$ trypsin inhibitor and $0.25 \%$ bovine albumin, $0.4 \% \mathrm{D}$ glucose, and 5\% fetal bovine serum (FBS; Gibco, ThermoFisher) in Minimum Essential Medium (MEM) w/Earl's Balanced Salt w/o L-glutamine ) at $37^{\circ} \mathrm{C}$ for $2 \mathrm{~min}$. After removing inhibition solutions and triturating dissociated cells with serum medium (0.4\% D-glucose and 5\% FBS in MEM w/Earl's Balanced Salt w/o L-glutamine), cells were seeded in 24-well plates with $\sim 80,000$ cells/well. Hippocampal cells were maintained in culture medium (Neurobasal ${ }^{\mathrm{TM}}-\mathrm{A}$ medium with serum-free B2 $7^{\mathrm{TM}}$ Supplement and GlutaMAX ${ }^{\mathrm{TM}} ;$ Gibco $\left.^{\mathrm{TM}}\right)$. Glial cells were inhibited by adding mitotic inhibitor ( $4 \mu \mathrm{M}$ 5-fluoro-2'-deoxyuridine and $10 \mu \mathrm{M}$ uridine in culture medium) into hippocampal culture at 3 days in vitro (DIV). All the imaging and stimulation are performed at $7-$ 


\section{DIV.}

HEK-293 cell culture and TRPV4 transfection. The HEK-293FT cell line was a gift from the lab of Feng Zhang (MIT). Cells were cultured in Dulbecco's Modified Eagle Medium (DMEM) w/ GlutaMAX ${ }^{\mathrm{TM}}$ supplemented with 10\% FBS. For imaging, HEK cells were cultured on $12 \mathrm{~mm}$ round coverslips which were coated with Matrigel® Matrix solution (Corning; Matrigel diluted 30x in DMEM with 10\% FBS) overnight prior to cell seeding in 24-well plate. When cells reach $70 \%$ confluency, transfection was performed by adding a mixture of $1 \mu$ l Lipofectamine ${ }^{\circledR} 2000$ and $0.5 \mu \mathrm{g}$ of sDNA plasmid (pLenti-CamKII $\alpha:$ TRPV4-p2A-mCherry) in $50 \mu 1$ of Opti-MEM. Cells were used for imaging after 24-48 hours of transfection.

Magnetomechanical stimulation. Magnetomechanical stimulation was evaluated by performing $\mathrm{Ca}^{2+}$ imaging on DRG explant culture, controlling hippocampal neurons and HEK cells decorated with MNDs and applying low frequency MF in situ. $1 \mathrm{mM}$ Fluo-4 AM (Invitrogen) in DMSO (Sigma-Aldrich) was diluted 1000x in Neurobasal ${ }^{\mathrm{TM}}$ medium with serum-free B2 ${ }^{\mathrm{TM}}$ supplement for DRG and hippocampal neurons. For HEK-293 cell culture, DMEM with 10\% FBS was used. Cells were incubated in this solution for 45 minutes and then transferred to Tyrode's solution. $30 \mu \mathrm{L}$ of magnetite nanodiscs at a concentration of $2 \mathrm{mg} / \mathrm{mL}$ were added to each well and the 24well plate was gently shaken to disperse magnetitic nanodiscs. After 15 minutes of incubation, DRGs on were taken from the 24 well plate, excess magnetite nanodiscs were washed off and coverslips with DRGs were added to a custom imaging chamber surrounded by a solenoid on an inverted microscope and, after a 5 min equilibration time, $\mathrm{Ca}^{2+}$ imaging began. During the experiment, slowly varying MFs were applied in $10 \mathrm{~s}$ pulses at intervals of $30 \mathrm{~s}$.

Pharmacology experiments. The magnetomechanical stimulation and $\mathrm{Ca}^{2+}$ imaging experiment described above was modified by adding drugs. The PIEZO-specific inhibitor GsMTx4 (Smartox) was added to Tyrode's solution at a concentration of $1 \mu \mathrm{M} .1 \mu \mathrm{M}$ of the TRPV4 antagonist HC067047 (MilliporeSigma) was added to the DRGs and HEK-293 cells transfected with TRPV4 and $\mathrm{Ca}^{2+}$ imaging began after $25 \mathrm{~m}$ of incubation. During the experiments, slowly varying $\mathrm{MF}(5 \mathrm{~Hz}$, $26 \mathrm{mT}$ ) was applied in $10 \mathrm{~s}$ pulses at intervals of $30 \mathrm{~s}$.

Calcium imaging analysis. Videos of calcium activity were collected on an inverted Olympus IX73 microscope in the .vsi format using the Olympus cellSens software. The frame rate was $4 \mathrm{~Hz}$. The .vsi files were processed in ImageJ with the Bioformats package. The "Threshold", 
"Watershed", and "Analyze Particles" functions were used to delineate, separate, and circle cells automatically, respectively. The average grayscale value in the ROIs generated this way was exported in a .csv file and processed with MATLAB (MathWorks). A custom MATLAB script was used to convert the average grayscale value of each ROI over time into $\Delta \mathrm{F} / \mathrm{F}_{0}$ traces using the algorithm described in Box 1 of Jia et al., ${ }^{29}$ with $\tau_{0}=0.75 \mathrm{~s}, \tau_{1}=2.5 \mathrm{~s}$, and $\tau_{2}=7.5 \mathrm{~s}$, but with the modification that a central moving average and $\mathrm{F}_{0}$ calculation was used instead of a simple moving average. A second MATLAB script was used for choosing at random subset of 50 cells from each experiment and classifying them as responsive or non-responsive based on whether their $\Delta \mathrm{F} / \mathrm{F}_{0}$ trace ever exceeds 10 times the standard deviation of the signal during the period before MF is ever applied. Heatmaps displaying $\Delta \mathrm{F} / \mathrm{F}_{0}$ vs. time were generated in Mathematica (Wolfram).

Cell viability and biocompatibility assay. The Invitrogen LIVE/DEAD ${ }^{\circledR}$ Viability/Cytotoxicity kit was used to assess whether magnetomechanical stimulation or exposure to MNDs caused cytotoxicity. The kit uses calcein AM (green) to mark live cells and ethidium homodimer-1 (red) to mark dead cells. The stock solutions were both diluted in a single mixture containing $400 \mathrm{nM}$ of both dyes. Immediately before and 30 minutes after stimulation, DRGs were imaged with an inverted microscope, and images were processed with a MATLAB script: each region was imaged with both red and green filters; the relative abundance of green cells and red cells (by area) was tabulated for each region for each condition, with $100 \%$ live cells corresponding to all cell area being green and none being red.

The biocompatibility of magnetite nanodiscs was examined using the alamarBlue metabolic assay (Thermo Scientific). DRGs were incubated on day 0 with alamarBlue solution (1:10 in cell medium) for 4 hours followed by fluorescence intensity reading using a plate reader with $545 \mathrm{~nm}$ excitation and $590 \mathrm{~nm}$ emission detection. Magnetite nanodiscs at concentration of $0.8 \mathrm{mg} / \mathrm{ml}$ were then added to each well with DRGs and incubated at $37^{\circ} \mathrm{C}$ while monitoring the DRG growth. The alamarBlue assay was repeated as described after 24 hours, 4 days and 8 days of culture. Cell viability and proliferation rate compared to the day 0 baseline was evaluated.

Immunohistochemistry. DRGs were seeded on coverslips and, following a 2-week growth period, fixed for $20 \mathrm{~min}$ in $4 \%$ paraformaldehyde. After three washes with PBS, cells were permeabilized with $0.3 \%$ (vol/vol) Triton X-100 for 10 minutes and blocked with $2.5 \%$ donkey serum in PBS overnight at $4{ }^{\circ} \mathrm{C}$. Cells were incubated for 2 hours at room temperature with primary 
antibody (rabbit anti-Neurofilament 1:500 (Sigma N4142) and mouse anti-S100 1:500 (Sigma S2532)) with 2.5\% donkey serum in PBS. After 3 washes of the cells with PBS, incubation with matching secondary antibody in PBS was performed (donkey anti-rabbit Alexa Fluor 488 (Invitrogen A21206) and donkey anti-mouse Alexa Fluor 568 (Invitrogen A10037), respectively, 1:1000) for 2 hours and with DAPI (4'6-diamidino-2-phenylindole)(1:50,000) for another 15 minutes, and washed three times with PBS. Coverslips were mounted onto glass microscope slides using mounting solution (Fluoromount-G®, SouthernBiotech).

Statistical analysis. Statistical differences were determined using two-tailed student's t-tests assuming unequal variances with the Bonferroni-Holm correction for multiple comparisons. A MATLAB script written by David M. Groppe and downloaded from mathworks.com was used to implement the Bonferroni-Holm correction.

Crystal structure drawing. Crystal structures of hematite and magnetite were prepared using the VESTA structure analysis software. ${ }^{30}$ 